(C2008 IEEE. Personal use of this material is permitted. However, permission to reprint/republish this material for advertising or promotional purposes or for creating new collective works for resale or redistribution to servers or lists, or to reuse any copyrighted component of this work in other works must be obtained from the IEEE 


\title{
Volumetric Quantification of Atherosclerotic Plaque in CT Considering Partial Volume Effect
}

\author{
Jamshid Dehmeshki*, Member, IEEE, Xujiong Ye, Hamdan Amin, Maryam Abaei, XinYu Lin, and \\ Salah D. Qanadli
}

\begin{abstract}
Coronary artery calcification (CAC) is quantified based on a computed tomography (CT) scan image. A calcified region is identified. Modified expectation maximization (MEM) of a statistical model for the calcified and background material is used to estimate the partial calcium content of the voxels. The algorithm limits the region over which MEM is performed. By using MEM, the statistical properties of the model are iteratively updated based on the calculated resultant calcium distribution from the previous iteration. The estimated statistical properties are used to generate a map of the partial calcium content in the calcified region. The volume of calcium in the calcified region is determined based on the map. The experimental results on a cardiac phantom, scanned 90 times using 15 different protocols, demonstrate that the proposed method is less sensitive to partial volume effect and noise, with average error of $9.5 \%$ (standard deviation (SD) of 5-7 $\mathrm{mm}^{3}$ ) compared with $67 \%$ (SD of 3-20 $\mathrm{mm}^{3}$ ) for conventional techniques. The high reproducibility of the proposed method for 35 patients, scanned twice using the same protocol at a minimum interval of $10 \mathrm{~min}$, shows that the method provides 2-3 times lower interscan variation than conventional techniques.
\end{abstract}

Index Terms-Coronary artery calcification, expectation-maximization, partial volume effect, proportion map, volume measurement.

\section{INTRODUCTION}

C ORONARY artery disease (CAD) currently is the leading cause of death in humans [1], [2]. Calcification of the coronary vessel wall is regarded as a marker of advanced coronary atherosclerosis [3], [4]. Early identification of CAD in patients can reduce morbidity and/or mortality. One marker for CAD is coronary artery calcification (CAC). The presence of CAC indicates underlying CAD. Recent experimental investigations have suggested that calcifications in atherosclerotic lesions should be considered as an active process [5]. Increasing coronary calcifications indicates CAD progression. Evidence suggests that the calcium score has a significant predictive value for subsequent cardiac events in both symptomatic and asymptomatic patients [6], [7]. The amount of calcification correlates with the amount of plaque present [8]. Therefore, accurate identification of the calcium amounts in atherosclerotic plaque areas may allow effective treatment to prevent further progression of CAD.

Manuscript received February 27, 2006; revised August 8, 2006. Asterisk indicates corresponding author.

*J. Dehmeshki is with Medicsight plc, London, W1J 5AT, U.K.. (e-mail: j.dehmeshki@kingston.ac.k).

X. Ye, H. Amin, M. Abaei, and X. Lin are with Medicsight plc, London, W1J 5AT, U.K. (e-mail: xujiong.ye@medicsight.com).

S. D. Qanadli is with Department of Radiology, CHUV - University of Lausanne, CH-1000 Lausanne, Switzerland.

Digital Object Identifier 10.1109/TMI.2007.893344
Advances in computed tomography (CT) scanning techniques have provided a means for quantifying calcium in the coronary arteries.

Accuracy and reproducibility have become two of the main issues in the quantification of CAC. High reproducibility is very important for follow-up studies to track the patient's coronary calcification development [9]-[11]. One of the known methods of quantification of calcium in the coronary arteries is the Agatston method [12]. In this method, a threshold of 130 Hounsfield unit (HU) is applied to the CT image. Applying the threshold typically facilitates the identification of all voxels above the threshold as containing calcium. A scoring system is often used to rate the severity of the calcification, based on the number of voxels above the threshold multiplied by a weight based on the highest intensity within the calcification. For example, if the highest intensity is between 130 and $200 \mathrm{HU}$, then the weight is 1; if between 200 and $300 \mathrm{HU}$, the weight is 2; and if over 300 $\mathrm{HU}$, the weight is 3 . The values of the threshold and the weights are based on empirical studies of coronary scans, using electron beam computed tomography (EBCT), and the subsequent outcome for the patients.

Several reports have demonstrated that the Agatston scoring method is not suited for reliable and reproducible quantification of coronary calcified atherosclerosis plaque for multiple clinical purposes such as plaque progression assessment [13]. A critical weakness of this method is that the segmentation solely makes use of fixed voxel intensity as threshold without considering any complicated scenarios in CT imaging such as partial volume effects (PVE) and spatial information.

It can be seen that the weighting factor $F$ in the Agatston scoring method is chosen based on the maximum intensity. Obviously, the use of the maximum intensity without consideration of the spatial information is highly sensitive to noise which makes the method inaccurate and less reproducible. To solve this problem, alternative methods such as measurement of calcium volume and calcium mass have been proposed and are gradually gaining clinical acceptances [14]-[18] as means of greater precision and reproducibly in measuring calcified plaques. The volume of the calcium is estimated by multiplying the number of voxels above the threshold (e.g., $130 \mathrm{HU}$ ) by the volume of each voxel. The mass of the calcium may be estimated by weighting each voxel above the threshold (e.g., $130 \mathrm{HU}$ ) according to its intensity, and summing up the weights.

However, the above methods still suffer from considerable inter-scan variability. For example, any change of alignment between the scanner and the scanned object can affect the number of voxels which fall above the threshold and/or the maximum measured intensity within the calcification. Also, tests of phan- 


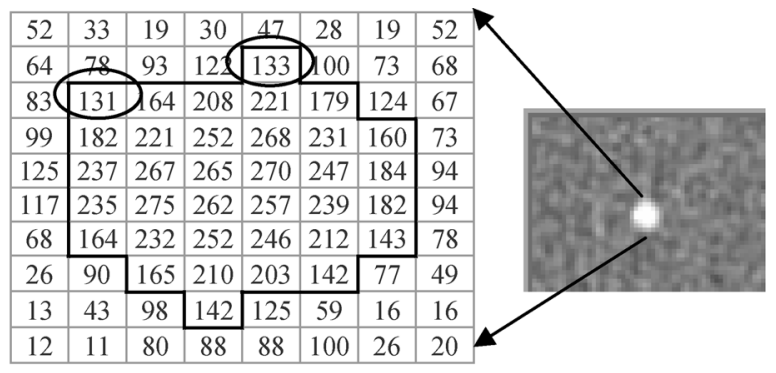

(a)

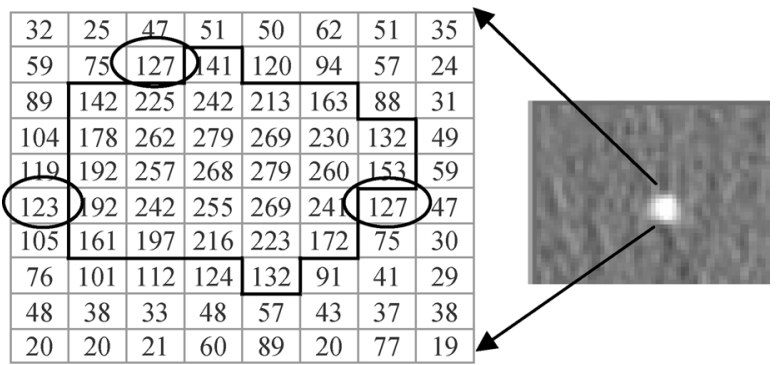

(b)

Fig. 1. Intensity maps for one calcified cylinder of QRM cardiac phantom scanned twice in (a) and (b).

toms (i.e., artificial objects having known properties) reveal that the methods are often inaccurate.

Therefore, there is a need for a method of calcium quantification that is more accurate, reproducible, and/or robust than such known techniques.

\section{BACKGROUND}

Due to the limited spatial resolution or motion artifacts of CT imaging, some voxels in cardiac CT images may contain a mixture of two or more tissue types instead of just a single tissue type. Theses voxels are referred as partial volume voxels (PVVs). The presence of the PVVs is significant especially when the structure is very tiny, e.g., small calcifications. Fig. 1 shows an example of one calcified region (C2) in QRM cardiac phantom (see Fig. 8). The true volume size of the cylinder is $21.2 \mathrm{~mm}^{3}$. The QRM phantom was scanned twice using different protocols. Fig. 1(a) is the intensity map of one cross section of the calcified cylinder from the first scanning and Fig. 1(b) is from the second scanning. The dot contours indicate the cylinder boundaries based on the thresholding of $130 \mathrm{HU}$. The cylinder volumetric size is defined as the total number of voxels in the calcium boundary multiplied by the voxel resolution. Based on the definition, the calculated cylinder volume is $28.31 \mathrm{~mm}^{3}$ in Scan 1 and $18.77 \mathrm{~mm}^{3}$ in Scan2. Compared to the true volume size of $21.2 \mathrm{~mm}^{3}$, the cylinder volume calculation for the first scan is an overestimation; while the calculation is an underestimation in the second scan. This is due to the PVE in CT imaging, especially on the boundary of the plaque. As can be seen from the intensity map shown in Fig. 1(a), the voxels on the dot contour could be the PVVs, especially the voxels with lower intensities (such as $133 \mathrm{HU}$ or $131 \mathrm{HU}$ ) might contain a very small proportion of the calcium component, and this is one of the reasons why the volume calculation using voxel based thresholding method is an overestimation for the first scan.
The same explanation relates to the volume underestimation of the same cylinder in the second scan. As seen in Fig. 1(b), along the cylinder boundary, voxels with intensities closer to $130 \mathrm{HU}$ (such as $127 \mathrm{HU}$ or $123 \mathrm{HU}$ ) may still contain a small proportion of the calcium component. So, by considering the calcium proportion component in each voxel rather than using the whole voxel, it can provide an accurate means of volume calculation.

The statistical models of PVE have been studied extensively in the literature [19]-[23]. Among these studies, the main objective of the method introduced in [19] was for object segmentation (such as calcium) rather than quantification. In this paper, an extention of this method is employed not only to detect the coronary calcium region, but also to quantify the calcium amount.

More specifically, a global statistical model is built to estimate the distributions of calcium and noncalcium (blood vessel), taking into account information from neighbouring voxels. A mixture of tissues statistic models are then defined considering spatial information for PVV calculations in which a maximum probability method is used to obtain the optimum proportion of the calcium component in each detected calcium voxel. Our comparison results show that the accuracy and reproducibility of the proposed method are superior to the voxel based $130 \mathrm{HU}$ thresholding method using 16-slice multidetector row CT (MDCT) in both of the cardiac phantom and real patients' data. The high reproducibility of the proposed method for the patient data particularly demonstrates the effectiveness of the new method to deal with motion artifacts.

\section{QUANTIFICATION OF CALCIFIED REGION}

A partial voxel based segmentation method is presented for accurate detection and quantification of CAC from CT images. This method processes the CT image to identify calcified areas. The method selects a 3-D calcified region and excludes any other calcified regions not forming part of the selected calcified region. The region is selected by applying a threshold (130 $\mathrm{HU}$ ), identifying connected regions that exceed the threshold, and then selecting one of the connected regions as the region for which calcification is to be quantified. Statistical parameters, such as mean and standard deviation of intensity, are calculated both for the selected calcified region and the noncalcified background. Modified expectation maximization (MEM) algorithm is used to calculate the statistical parameters. The MEM algorithm is applied iteratively and can be performed until the estimated statistical parameters converge to a predetermined degree between successive iterations. Based on the estimated statistical parameters, the estimated partial content of calcium per voxel is calculated in the calcified region. The estimated partial content values are processed to generate a map of partial volume of calcium in the selected calcified region. The volume of calcium in the calcified region is determined based on the map Fig. 2 provides a flow diagram outlining the above key steps.

\section{A. Region Identification}

The region identification is performed by the thresholding method to segment the image into foreground and background. The foreground areas are grouped into one or more calcified regions. If more than one region is found, the method selects a 


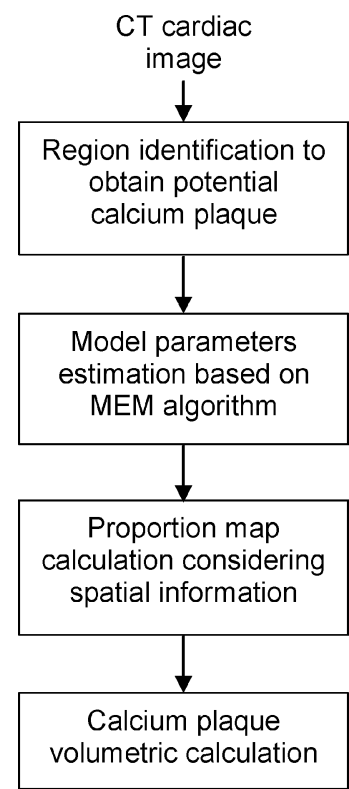

Fig. 2. Flow diagram of the proposed statistic based partial voxel method.

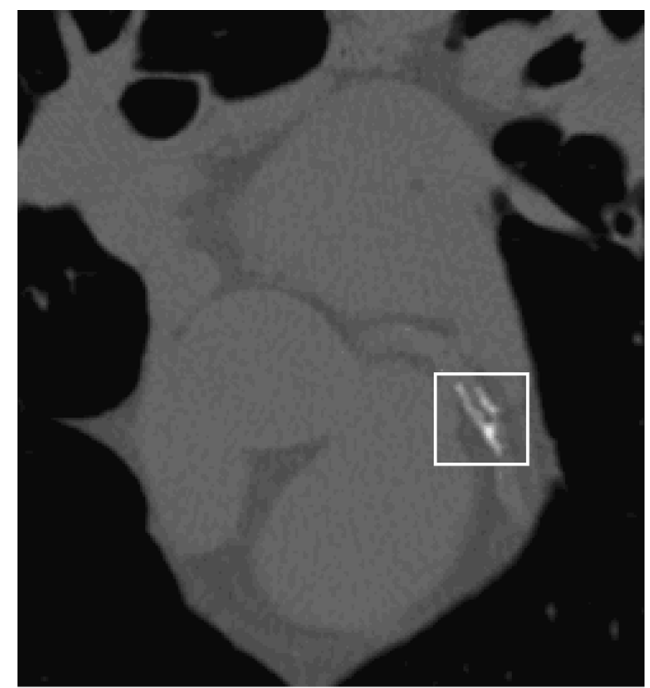

(a)

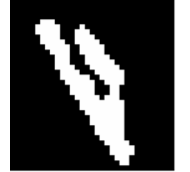

(b)

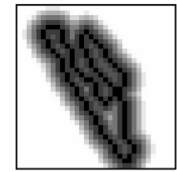

(c)

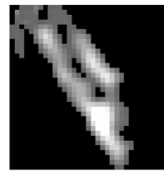

(d)
Fig. 3. Coarse segmentation of calcium lesion. (a) Original CT image. (b) Segmented region based on the $130 \mathrm{HU}$ thresholding method. (c) Distance transform map of (b). (d) Subimage of (a) based on enlarged region mask obtained from distance transform.

region and defines an enlarged region including a background area around the selected region but excluding nonselected regions.

Fig. 3 illustrates a calcified region in a scan image. A predetermined threshold (e.g., $130 \mathrm{HU}$ ) is applied to each voxel in the image. The voxels having an intensity that exceeds the threshold are referred to as foreground, and the remainder as background. The foreground areas are grouped into one or more discrete regions using a simple 3-D binary region-growing or 3-D labeling technique (based on 26-connectivity of the propagation process) [24].

A calcified region is initially defined as containing only the seed point. Region growing is performed by iteratively adding adjacent or neighboring foreground voxels to the region until there are no more foreground voxels adjacent to the region.

If more than one region is found in the image, further regions may be identified by applying the similar 26-connectivity region-growing from other seed points not belonging to any of the regions already found. Fig. 3(b) shows one identified calcified region.

The region is then relatively enlarged using a distance transform technique to obtain an enlarged region. For each identified calcified region, the distance transform map is calculated and the maximum distance of the region related to region boundary is obtained. The enlarged region is then defined as $s * f \max$, where $\mathrm{s}$ is the enlarged factor, fmax is the maximum distance value of the region in the distance transform map. In this study, $s=2.0$. Fig. 3(c) is the distance transform map of (b).

Foreground voxels not forming part of the original region are removed from the enlarged region to obtain a final region. This final region is used as a mask to define the maximum potential extent of the calcified region. Fig. 3(d) shows the subimage obtained from overlapping the enlarged mask on the original image (a). Voxels outside this region are not taken into account when estimating the extent and/or properties of the calcified region.

\section{B. MEM Algorithm}

MEM algorithm [19] can be used iteratively to estimate the probability that each voxel in the enlarged region mask [e.g., Fig. 3(d)] represents calcium. A statistical model can be constructed to estimate such parameters based on the MEM algorithm. An intensity image $Y=\left\{y_{i}, i=1,2, \ldots, I\right\}$ of region mask with $I$ voxels of intensity $y_{i}$ and $K$ different classes, $\hat{L}=$ $\{1,2, \ldots, K\}$, is provided. A special case includes two classes or tissue types: calcium and noncalcium (i.e., blood vessel).

It is noted that the use of a threshold of $130 \mathrm{HU}$ in Agatston method [12] to identify calcifications is based on the density of two standard deviations above the average density of blood in the aorta. Due to the above fact and by simplifying the model, the ranges of image intensities corresponding to the non-calcium (blood vessel) and calcium are modelled as Gaussian distributions.

Image intensity in CT imaging is spatially dependent. For instance, voxels with the same intensity may have different structural properties. A mixed statistical model that considers spatial properties is employed for the distribution of voxel intensity $p\left(y_{i} \mid \vartheta\right)$ as follows:

$$
p\left(y_{i} \mid \vartheta\right)=\sum_{l \in \hat{L}} a_{l}(i) \cdot p_{l}\left(y_{i} \mid \varphi_{l}\right) \quad i=1,2, \ldots, I
$$

where, for each $i$

$$
p_{l}\left(y_{i} \mid \varphi_{l}\right)=G\left(\mu_{l}, \sigma_{l}\right)=\frac{1}{\sqrt{2 \pi} \sigma_{l}} \exp \left(-\frac{\left(y_{i}-\mu_{l}\right)^{2}}{2 \sigma_{l}^{2}}\right)
$$

which is a Gaussian distribution with parameters $\phi_{l}=\left(\mu_{l}, \sigma_{l}\right)$ and $\vartheta=\left(a_{1}(i), a_{2}(i), \ldots, a_{K}(i), \varphi_{1}, \varphi_{2}, \ldots, \varphi_{K}\right) . a_{l}(i)$ is a spatial prior probability with spatial constraints imposed by 
a Markov random field (MRF) and Gibbs random field (MRFGRF) [25].

A MRF $F=\left\{F_{1}, F_{2}, \ldots, F_{k}\right\}$ defined on the set $S$ is a lattice indexing the voxels in the given image $Y$, in which each random variable $F_{i}$ takes a value $l_{i} \in \hat{L}$. The probability density of the MRF $F$ can be given by the Gibbs distribution

$$
p(F)=Z^{-1} \exp [-U(F)]
$$

where $U(F)=\sum_{c \in C} v_{c}(F)$ is the energy function. The energy function is a sum of clique potentials $v_{c}(F)$ over all possible cliques in the enlarged region mask, and $Z$ is a normalization term. According to the Hammersley Clifford theorem, the conditional probability can be derived using MRF-GRF equivalence as follows:

$$
p\left(l_{i} \mid l_{N(i)}\right)=\frac{\exp \left[-\sum v_{c}\left(l_{i}\right)\right]}{\sum_{l_{i} \in \hat{L}} \exp \left[-\sum v_{c}\left(l_{i}\right)\right]}
$$

where $N(i)$ is the neighbourhood of voxel $i$.

Assuming the spatial prior distribution $a_{l}(i)$ in (1) is given by the MRF conditional probability $p\left(l_{i} \mid l_{N(i)}\right)$ in (2), according to the Bayesian probability theory, the posterior probability $p\left(\varphi_{i} \mid y_{i}\right)$ can be obtained as

$$
p\left(\varphi_{l} \mid y_{i}\right)=\frac{p_{l}\left(y_{i} \mid \varphi_{l}\right) \cdot p\left(l_{i} \mid l_{N(i)}\right)}{p\left(y_{i} \mid \vartheta\right)} .
$$

Here, the potential function in (2) is defined as

$$
v_{c}\left(l_{i}\right)=\beta \cdot p\left(\varphi_{l} \mid y_{j}\right), \quad j \in c
$$

where $\beta$ is a positive constant which controls the size of clustering. The posterior probability $p\left(\varphi_{l} \mid y_{i}\right)$ represents the probability that the given voxel $i$ belongs to one class $l_{i} \in \hat{L}$. Equation (3) can be used to estimate the highest probability of the reconstructed label image $L$ based on the observed intensity value and the image model as defined in (1) and (2). The model parameters $\vartheta$ can be obtained to solve (3).

To adapt the model defined in (1) and (2) so that the spatial information is considered by using MRF-GRF model, a modified version of the two-step EM algorithm (i.e., an MEM algorithm) may be used to estimate parameters of the model and classify voxels of each group simultaneously. For example, for a given $\phi_{l}^{m}=\left(\mu_{l}^{m}, \sigma_{l}^{m}\right)$, the unique solution $\phi_{l}^{m+1}=\left(\mu_{l}^{m+1}, \sigma_{l}^{m+1}\right)$ can be derived as

$$
\begin{aligned}
\mu_{l}^{m+1} & =\frac{\left\{\sum_{i=1}^{I} y_{i} \frac{a_{l}^{m}(i) p\left(y_{i} \mid \varphi_{l}^{m}\right)}{p\left(y_{i} \mid \vartheta^{m}\right)}\right\}}{\sum_{i=1}^{I} \frac{a_{l}^{m}(i) p\left(y_{i} \mid \varphi_{l}^{m}\right)}{p\left(y_{i} \mid \vartheta^{m}\right)}} \\
\sigma_{l}^{2(m+1)} & =\frac{\left\{\sum_{i=1}^{I}\left(y_{i}-\mu_{l}^{m+1}\right)^{2} \frac{a_{l}^{m}(i) p\left(y_{i} \mid \varphi_{l}^{m}\right)}{p\left(y_{i} \mid \vartheta^{m}\right)}\right\}}{\sum_{i=1}^{I} \frac{a_{l}^{m}(i) p\left(y_{i} \mid \varphi_{l}^{m}\right)}{p\left(y_{i} \mid \vartheta^{m}\right)}}
\end{aligned}
$$

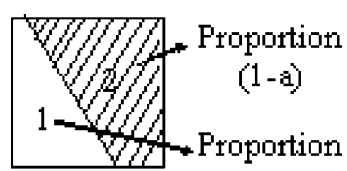

(a)

Fig. 4. One voxel containing calcium and blood.

where $a_{l}^{m}$ in each step can be approximately calculated by assuming

$$
a_{l}^{m}(i) \approx p\left(l_{i} \mid l_{N(i)}\right)=\frac{\exp \left[-\sum v_{c}\left(l_{i}\right)\right]}{\sum_{l_{i} \in \hat{L}} \exp \left[-\sum v_{c}\left(l_{i}\right)\right]} .
$$

The process converges after sufficient iterations, and may be halted after a predetermined number of iterations and/or once a predetermined convergence criterion is met.

The MEM algorithm iteratively calculates a statistical classification of the voxels based on the model parameters of the previous iteration and updates the parameters accordingly. The use of MRF-GRF as a spatial constraint can improve the voxelbased image classification performance of the MEM algorithm, especially in the presence of noisy image data.

\section{Estimate of Partial Content of Calcium}

MEM algorithm is used to estimate the statistical model parameters in (5) and to detect the calcium region by taking into account PVE problem. In this section, the estimated model parameters are employed to calculate the proportion of calcium in "partial voxels," which are a mixture of calcium and noncalcium (blood vessel) (see Fig. 4).

A model of calcified material with probability distribution of $p_{1}\left(\mu_{1}, \sigma_{1}\right)$, and a model of non-calcified material with probability distribution of $p_{2}\left(\mu_{2}, \sigma_{2}\right)$, are associated with a particular voxel, where $\mu$ and $\sigma$ are the mean and standard deviation of the Gaussian model. These parameters can be estimated using MEM [(5)]. The distribution for the combined intensities follows a linear combination of two Gaussians

$p\left(y_{i} \mid a^{i}\right)=G\left(a^{i} \mu_{1}+\left(1-a^{i}\right) \mu_{2}, \sqrt{a^{i} \sigma_{1}^{2}+\left(1-a^{i}\right) \sigma_{2}^{2}}\right)$

where $a^{i}$ is the proportion of calcium in voxel $i$, and the proportion of noncalcium (blood vessel) in the voxel is $\left(1-a^{i}\right)$.

According to Bayes' theorem, given voxel $i$, the statistical distribution of the proportion of calcium component in this voxel can be calculated using the following equation:

$$
p\left(a^{i} \mid y_{i}\right)=\frac{p\left(y_{i} \mid a^{i}\right) \cdot p\left(a^{i}\right)}{p\left(y_{i}\right)}
$$

where $p\left(y_{i}\right)$ is a normalizing constant, and $p\left(a^{i}\right)$ is the prior probability of the $i$ th voxel having proportion $a$ of calcium. $p\left(a^{i}\right)$ can be calculated in at least two different ways.

Method 1: Assuming the prior probability is modelled as a uniform distribution in the range [0,1], i.e., $p\left(a^{i}\right)=1, \forall i$. According to an embodiment, only partial voxels are considered to determine $p\left(a^{i} \mid y_{i}\right)$. The profiles of $p\left(a^{i} \mid y_{i}\right)$ at different 

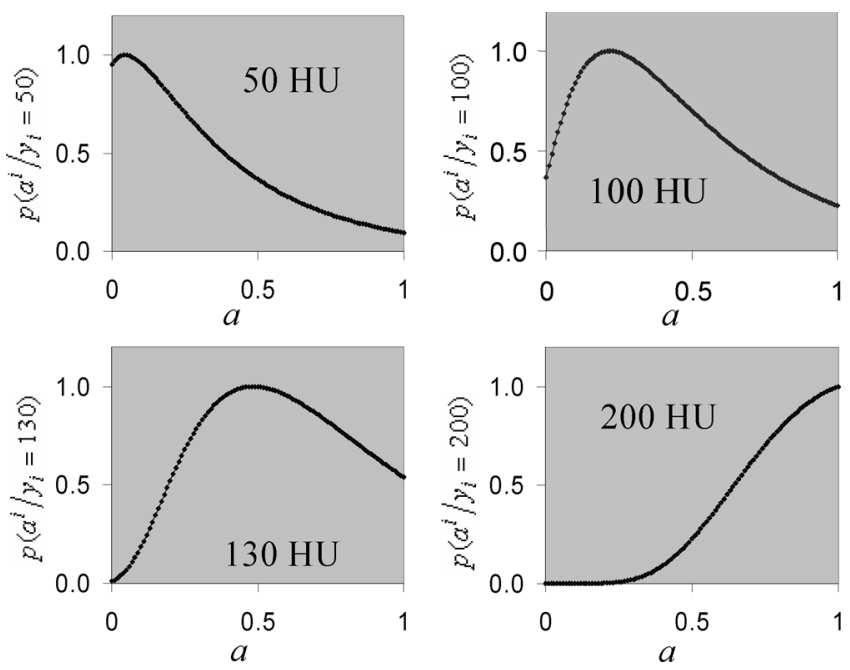

Fig. 5. Profiles of $p\left(a^{i} \mid y_{i}\right)$ at different intensities $\left(y_{i}=50 \mathrm{HU}, 100 \mathrm{HU}, 130\right.$ HU, $200 \mathrm{HU}$ ) generated based on (6) using a typical real CT subimage.

intensity values $y_{i}$ are calculated based on the estimated statistical values. Fig. 5 shows profiles of $p\left(a^{i} \mid y_{i}\right)$ at four different exemplary intensity values $\left(y_{i}=50 \mathrm{HU}, 100 \mathrm{HU}, 130 \mathrm{HU}\right.$, and $200 \mathrm{HU})$ of a CT image. In each profile in Fig. 5, the horizontal axis represents the proportion of calcium in the voxel, and the vertical axis represents the probability of the voxel containing the proportion $a$ of calcium.

Method 2: $p\left(a^{i}\right)$ (MRF) can be based on the neighbourhood of a voxel. In (6), the initial prior probability $p\left(a^{i}\right)$ is updated based on $p\left(a^{i} \mid y_{i}\right)$ of the neighbourhood. Only neighbouring sites have direct interactions with each other, and they tend to have the same class labels. Based on a technique related to the Gibbs distributions, the prior probability can be derived as follows:

$$
p\left(a^{i}\right)=\frac{\exp \left[-\sum v_{c}(a)\right]}{\sum_{a} \exp \left[-\sum v_{c}(a)\right]}
$$

where $v_{c}(a)=\beta \cdot p\left(a^{i} \mid y_{i}\right), s \in c$, over all possible cliques c. An exemplary set of conditions may be as follows: $a=0.1 \times k$, $k \in[0,10]$, and $\beta=0.5$

In this study, method 2 was employed. To calculate the amount of a certain calcium in one voxel, one can determine the highest probability of $a$, namely

$$
a_{\text {optimal }}^{i}=\max _{a} P\left(a^{i} \mid y_{i}\right)
$$

Hence the total volumetric size of the calcium plaque is defined as

$$
V=V_{\text {voxel }} * \sum_{i}\left(a_{\text {optimal }}^{i}\right)
$$

where $V_{\text {voxel }}$ is the volume of one voxel.

Fig. 6 shows an example of the calcium plaque and its corresponding proportion map. Fig. 6(a) is the original calcium plaque subimage while Fig. 6(c) is the corresponding HU values of voxels containing the calcium plaque. The potential

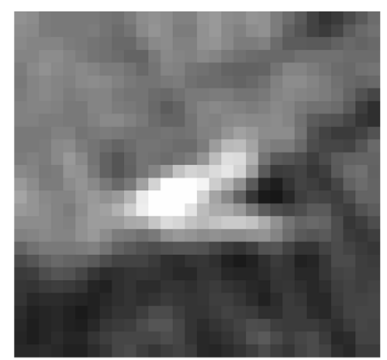

(a) Original image

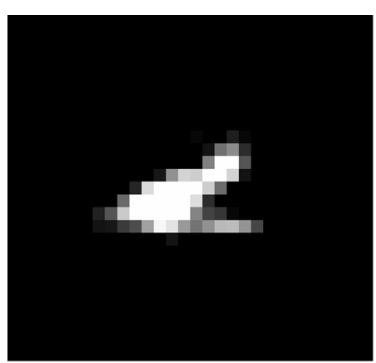

(b) Proportion map

\begin{tabular}{|c|c|c|c|c|c|c|c|c|c|c|c|c|c|}
\hline 40 & 34 & 28 & 20 & 20 & 24 & 18 & 24 & 42 & 52 & 74 & 92 & 80 & 60 \\
\hline 14 & 2 & 10 & 24 & 24 & 20 & 12 & 10 & 36 & 96 & 130 & 140 & 100 & 50 \\
\hline-6 & -22 & 2 & 28 & 20 & 14 & 22 & 38 & 74 & 136 & 188 & 184 & 118 & 28 \\
\hline-4 & -24 & -6 & 28 & 52 & 88 & 142 & 168 & 166 & 184 & 200 & 168 & 86 & -16 \\
\hline 31 & 8 & 32 & 96 & 170 & 248 & 300 & 286 & 220 & 166 & 128 & 70 & -20 & -100 \\
\hline 70 & 79 & 126 & 206 & 290 & $\mathbf{3 3 6}$ & 330 & 268 & 172 & 100 & 58 & 0 & -76 & -128 \\
\hline 94 & 117 & 164 & 216 & 270 & 288 & 250 & 192 & 138 & 110 & 104 & 80 & 32 & -10 \\
\hline 86 & 88 & 105 & \multicolumn{114}{|c|}{} & 148 & 192 & 174 & 146 & 132 & 142 & 158 & 160 & 144 & 112 \\
\hline 62 & 39 & 30 & 16 & 32 & 72 & 84 & 74 & 64 & 66 & 64 & 62 & 72 & 76 \\
\hline
\end{tabular}

(c) Intensity value of the calcium for each voxel

\begin{tabular}{|l|l|l|l|l|l|l|l|l|l|l|l|l|l|}
0.00 & 0.00 & 0.00 & 0.00 & 0.00 & 0.00 & 0.00 & 0.00 & 0.02 & 0.00 & 0.00 & 0.10 & 0.02 & 0.00
\end{tabular} $\begin{array}{llllllllllllllll}0.00 & 0.00 & 0.00 & 0.00 & 0.00 & 0.00 & 0.00 & 0.00 & 0.00 & 0.06 & 0.45 & 0.55 & 0.17 & 0.00\end{array}$ $\begin{array}{llllllllllllll}0.00 & 0.00 & 0.00 & 0.00 & 0.00 & 0.00 & 0.00 & 0.00 & 0.00 & 0.51 & 1.00 & 0.99 & 0.34 & 0.00\end{array}$ \begin{tabular}{lllllll|l|l|l|l|l|l|l|}
0.00 & 0.00 & 0.00 & 0.00 & 0.00 & 0.07 & 0.57 & 0.83 & 0.81 & 0.99 & 1.00 & 0.83 & 0.06 & 0.00
\end{tabular} \begin{tabular}{lll|l|l|l|l|l|l|l|l|l|l|l}
0.00 & 0.00 & 0.00 & 0.14 & 0.85 & 1.00 & 1.00 & 1.00 & 1.00 & 0.81 & 0.43 & 0.00 & 0.00 & 0.00
\end{tabular}

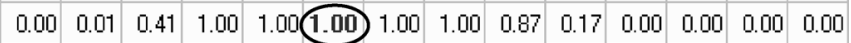
$\begin{array}{llllllllllllllll}0.12 & 0.33 & 0.79 & 1.00 & 1.00 & 1.00 & 1.00 & 1.00 & 0.53 & 0.26 & 0.21 & 0.02 & 0.00 & 0.00\end{array}$

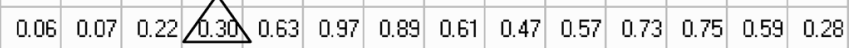
$\begin{array}{llllllllllllllll}0.00 & 0.00 & 0.00 & 0.00 & 0.00 & 0.00 & 0.05 & 0.00 & 0.00 & 0.00 & 0.00 & 0.00 & 0.00 & 0.00\end{array}$

(d) Proportion value of the calcium for each voxel

Fig. 6. Typical example of the proportion map for a calcium plaque.

calcified region is initially identified using the algorithm described in Section III-A. The MEM algorithm is then applied on the enlarged coarse region to calculate each class parameters $\varphi_{l}\left(\mu_{l}, \sigma_{l}\right)$ based on (5). Next, the proportion of the calcium at each voxel is calculated by using (8). Fig. 6(b) is the calculated proportion map and Fig. 6(d) is the proportion values of voxels in the proportion map which corresponds to the intensity values shown in Fig. 6(c). From Fig. 6(c) and (d), it can be seen that the proportion values in the core part of the calcium area (HU values over $188 \mathrm{HU}$ ) are 1 signifying that those voxels contain only a calcium component; while the proportion values on the surrounding area are less than 1 (such as 0.30 for the voxel with $114 \mathrm{HU}$ ) meaning that those voxels only contain a partial calcium component.

\section{EXPERIMENTAL RESULTS}

Two studies were conducted to compare the accuracy and reproducibility of the proposed method with traditional one that was described earlier. In the first study, a cardiac phantom (QRM, Moehrendorf, Germany) study was performed to show how the proposed method gives accurate volume measurements under different intensity contrasts and radiation doses. Considering the motion artifact, in the second study, reproducibility was further evaluated in 35 patients who were scanned twice 


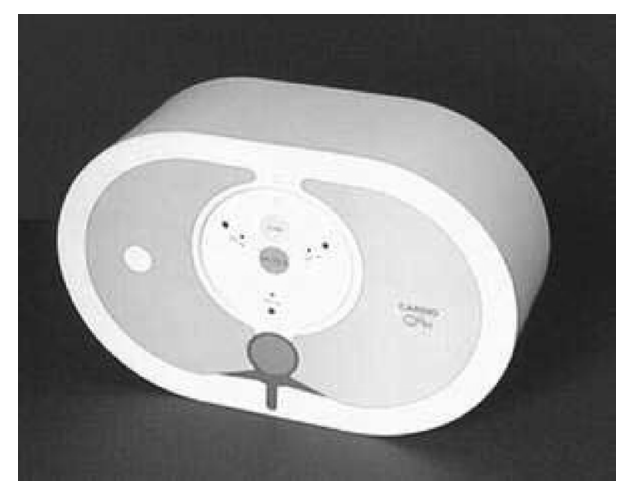

Fig. 7. QRM cardiac phantom.

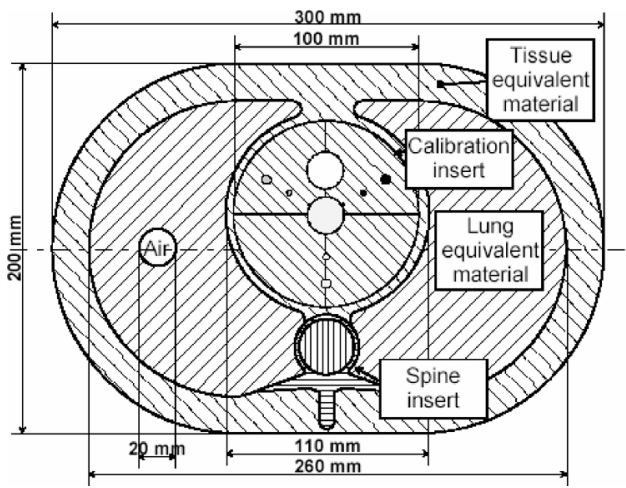

Fig. 8. Sketch of the phantom body.

using the same protocol at a minimum interval of $10 \mathrm{~min}$. The standard deviation (std) of the total calcium volume size between two series was calculated to demonstrate the effectiveness of the proposed method in the presence of motion artifacts. The following are the results of experiments.

\section{A. QRM Cardiac Phantom Study}

The cardiac phantom consists of two parts: an anthropomorphic phantom body and heart calibration insert (Fig. 7). The phantom body contains artificial lungs and a spine insert surrounded by soft tissue equivalent material chosen with respect to real attenuation characteristics of X-ray in the thorax. At the anatomic position of the heart is a cylindrical hole of $100 \mathrm{~mm}$ diameter which encompasses the heart calibration insert. Fig. 8 shows a sketch of the phantom body with the calibration insert. The calibration insert itself contains nine cylindrical objects that vary in size and hydroxyapatite density which is organized on three series. Each of the series contains three different sizes being 1,3 , and $5 \mathrm{~mm}$ and three different densities of hydroxyapatite (CaHA) that are 200, 400, and $800 \mathrm{mg} / \mathrm{cm}^{3}$.

The phantom was scanned 90 times on a 16-slice Multi-Detector Row CT (MDCT) (GE LightSpeed Pro) using 15 different protocols. Acquisition protocols were chosen to provide a large spectra of acquisition parameters that simulate different clinical situations to minimize and maximize the partial volume effect and vary the noise level (Table I). The phantom was placed in three different angular positions $\left(0^{\circ}, 45^{\circ}\right.$, and $\left.90^{\circ}\right)$ to the $z$-axis and each protocol was used to scan the phantom twice. A
TABLE I

15 CT Protocol SETtings on Phantom Study

\begin{tabular}{c|c|c}
\hline $\begin{array}{c}\text { Slice Thickness } \\
(\mathbf{m m})\end{array}$ & $\begin{array}{c}\text { Tube Current } \\
(\mathbf{m A})\end{array}$ & $\begin{array}{c}\text { Beam Collimator } \\
(\mathbf{m m})\end{array}$ \\
\hline 1.25 & 300 & 10 \\
\hline 2.5 & 300 & 10 \\
\hline 1.25 & 250 & 10 \\
\hline 2.5 & 250 & 10 \\
\hline 1.25 & 200 & 10 \\
\hline 2.5 & 200 & 10 \\
\hline 1.25 & 150 & 10 \\
\hline 2.5 & 150 & 10 \\
\hline 1.25 & 100 & 10 \\
\hline 2.5 & 100 & 10 \\
\hline 2.5 & 300 & 20 \\
\hline 2.5 & 250 & 20 \\
\hline 2.5 & 200 & 20 \\
\hline 2.5 & 150 & 20 \\
\hline 2.5 & 100 & 20 \\
\hline
\end{tabular}

sample slice containing six simulated calcium plaques is shown in Fig. 9(a). Note that in this study, only the calcium insets with a diameter larger than $1 \mathrm{~mm}$ are considered. The plaques had three different $\mathrm{HU}$ of about $270 \mathrm{HU}, 550 \mathrm{HU}$, and $1100 \mathrm{HU}$.The actual or true values for the plaques in the phantom are given in Table II.

Fig. 9(c) and (d) clearly highlights the dramatic difference between the proposed and conventional methods. Fig. 9(c) is the proportion map of one calcium plaque $(\mathrm{C} 2)$ using the proposed method while Fig. 9(d) is the same calcified region detected by the conventional method. It is observed that in the proposed method shown in Fig. 9(c), some voxels near the plaque boundary have values less than 1.0, indicating a mixture of materials (calcium and noncalcium), the number being the fraction of calcium. However, in the conventional method shown in Fig. 9(d) those voxels are incorrectly labeled as $100 \%$ calcium illustrating a tendency to overestimate the area.

The tendency to overestimate the volume as shown in Fig. 9(c) and (d) is also apparent when averaged over 90 scans as shown in Table II. Volume measurements using the proposed method have a low bias or systematic error (the difference between true value and mean volume calculated by the proposed method is about $1.1-5.6 \mathrm{~mm}^{3}$, the average error of $9.5 \%$ ), which is almost independent of the plaque size and density. In contrast, the conventional method has larger systematic errors (up to 86 $\mathrm{mm}^{3}$, with an average error of $67 \%$ ) that depend on both plaque size and density. Fig. 10 shows the relationship of the accuracy of the plaque averaged volume measurements over 90 scans with the plaque size and density for the two methods.

For the reproducibility, the proposed partial voxel based method demonstrates reasonably good reproducibility, with a standard deviation of about $4.1-6.3 \mathrm{~mm}^{3}$, regardless of plaque size and density. In contrast, the conventional method is less reproducible for all plaques $\left(3.1-19.7 \mathrm{~mm}^{3}\right)$. Fig. 11 shows the relationship of the reproducibility of the plaque averaged volume measurements over 90 scans with the plaque size and density for different methods.

Fig. 12 shows a comparative result of volume measurements for plaque A1 using the two methods. It can be seen that the pro- 


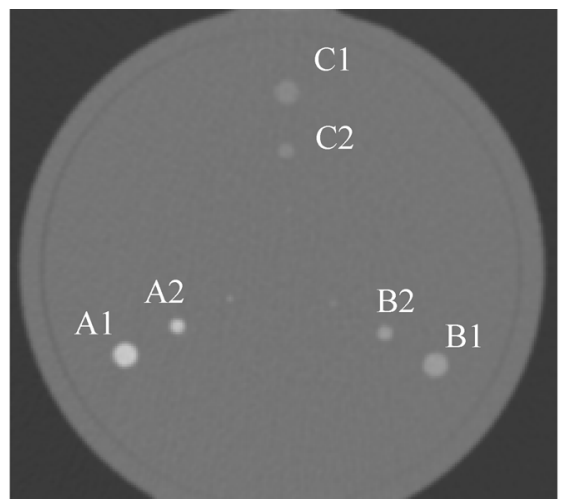

(a) One phantom slice containing objects (plaques) with two different sizes and three different contrasts

\begin{tabular}{|c|c|c|c|c|c|c|c|c|c|}
\hline 40 & 51 & 47 & 38 & 31 & 40 & 46 & 35 & 35 & 44 \\
\hline 40 & 55 & 82 & 109 & 108 & 107 & 96 & 54 & 30 & 41 \\
\hline 35 & 76 & 143 & 200 & 205 & 191 & 154 & 80 & 29 & 35 \\
\hline 48 & 124 & 207 & 251 & 253 & 253 & 218 & 127 & 52 & 36 \\
\hline 62 & 164 & 248 & 267 & 259 & 274 & 264 & 174 & 85 & 49 \\
\hline 69 & 178 & 267 & 284 & 262 & 264 & 257 & 182 & 94 & 55 \\
\hline 58 & 151 & 238 & 273 & 256 & 235 & 211 & 148 & 76 & 40 \\
\hline 45 & 92 & 149 & 186 & 192 & 179 & 148 & 96 & 51 & 27 \\
\hline 41 & 44 & 56 & 67 & 79 & 93 & 78 & 46 & 37 & 42 \\
\hline 46 & 41 & 33 & 11 & 3 & 21 & 23 & 16 & 45 & 70 \\
\hline
\end{tabular}

(b) HU Value of plaque C2

\begin{tabular}{|l|l|l|l|l|l|l|l|l|l|}
\hline 0.00 & 0.00 & 0.00 & 0.00 & 0.00 & 0.00 & 0.00 & 0.00 & 0.00 & 0.00 \\
\hline 0.00 & 0.00 & 0.06 & 0.30 & 0.29 & 0.28 & 0.18 & 0.00 & 0.00 & 0.00 \\
\hline 0.00 & 0.01 & 0.60 & 1.00 & 1.00 & 1.00 & 0.70 & 0.04 & 0.00 & 0.00 \\
\hline 0.00 & 0.43 & 1.00 & 1.00 & 1.00 & 1.00 & 1.00 & 0.46 & 0.00 & 0.00 \\
\hline 0.00 & 0.79 & 1.00 & 1.00 & 1.00 & 1.00 & 1.00 & 0.88 & 0.09 & 0.00 \\
\hline 0.00 & 0.92 & 1.00 & 1.00 & 1.00 & 1.00 & 1.00 & 0.95 & 0.17 & 0.00 \\
\hline 0.00 & 0.67 & 1.00 & 1.00 & 1.00 & 1.00 & 1.00 & 0.65 & 0.01 & 0.00 \\
\hline 0.00 & 0.15 & 0.66 & 0.99 & 1.00 & 0.93 & 0.65 & 0.18 & 0.00 & 0.00 \\
\hline 0.00 & 0.00 & 0.00 & 0.00 & 0.04 & 0.16 & 0.03 & 0.00 & 0.00 & 0.00 \\
\hline 0.00 & 0.00 & 0.00 & 0.00 & 0.00 & 0.00 & 0.00 & 0.00 & 0.00 & 0.00 \\
\hline
\end{tabular}

(c) Proportion of calcium for each voxel of plaque $\mathrm{C} 2$ using proposed method

\begin{tabular}{|l|l|l|l|l|l|l|l|l|l|}
\hline 0 & 0 & 0 & 0 & 0 & 0 & 0 & 0 & 0 & 0 \\
\hline 0 & 0 & 0 & 0 & 0 & 0 & 0 & 0 & 0 & 0 \\
\hline 0 & 0 & 1 & 1 & 1 & 1 & 1 & 0 & 0 & 0 \\
\hline 0 & 0 & 1 & 1 & 1 & 1 & 1 & \multicolumn{1}{|c|}{} & 0 & 0 \\
\hline 0 & 1 & 1 & 1 & 1 & 1 & 1 & 1 & 0 & 0 \\
\hline 0 & 1 & 1 & 1 & 1 & 1 & 1 & 1 & 0 & 0 \\
\hline 0 & 1 & 1 & 1 & 1 & 1 & 1 & 1 & 0 & 0 \\
\hline 0 & 0 & 1 & 1 & 1 & 1 & 1 & 0 & 0 & 0 \\
\hline 0 & 0 & 0 & 0 & 0 & 0 & 0 & 0 & 0 & 0 \\
\hline 0 & 0 & 0 & 0 & 0 & 0 & 0 & 0 & 0 & 0 \\
\hline
\end{tabular}

(d) Calcified voxels of plaque $\mathrm{C} 2$ identified using conventional method: $1=$ calcium

Fig. 9. Comparative example of one calcium plaque on phantom using the proposed and conventional methods.

posed method on the stationary phantom gives superior results in terms of accuracy and reproducibility since the measured volumes are very much closer to the true values and there is less variation.
TABLE II

A Summary of the AVERAge Volume MeAsurements of 90 DifFERENT ACQUISITIONS USING THE TWO DIFFERENT METHODS (THE UNIT FOR VOLUME IS $\mathrm{mm}^{3}$ ); VOLUME (WITH PVE, PROPOSED METHOD); VoluME (Without PVE, CONVENTIONAL METHOD)

\begin{tabular}{lccccc}
\hline & $\begin{array}{c}\text { True } \\
\text { value }\end{array}$ & $\begin{array}{c}\text { Volume } \\
\text { (without PVE / } \\
\text { Conventional) }\end{array}$ & $\begin{array}{c}\text { Volume } \\
\text { (with PVE / Proposed) }\end{array}$ \\
\hline \hline plaque & volume & mean & std & mean & Std \\
\hline A1 & 98.2 & 184.2 & 19.7 & 103.8 & 5.7 \\
\hline A2 & 21.2 & 55.2 & 6.3 & 26.7 & 4.8 \\
\hline B1 & 98.2 & 148.0 & 13.9 & 101.0 & 6.3 \\
\hline B2 & 21.2 & 40.6 & 6.2 & 24.3 & 4.9 \\
\hline C1 & 98.2 & 105.5 & 5.5 & 95.2 & 4.6 \\
\hline C2 & 21.2 & 23.0 & 3.1 & 20.1 & 4.1 \\
\hline
\end{tabular}

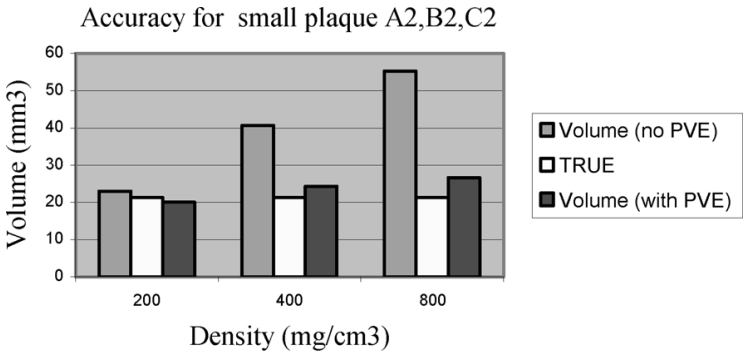

(a)

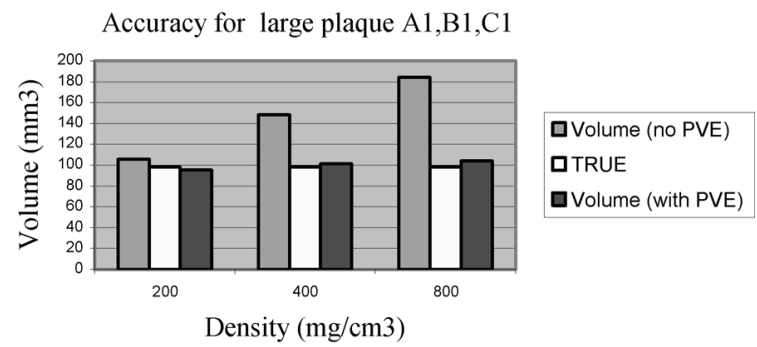

(b)

Fig. 10. Averaged volume measurements of 90 different acquisitions with three different densities for different size plaques. (a) Small plaques. (b) Large plaques.

\section{B. Real Clinical Data Study}

The in vivo experiments in this section aim to illustrate, with cardiac motion, how the proposed method gives high reproducibility for the volumetric measurements of CAC.

Thirty-five patients, with known or suspected ischemic heart disease or at risk of developing ischemic heart disease were enrolled in the study. Each patient underwent two unenhanced CT scans using the same parameters $(120 \mathrm{kV}, 200 \mathrm{~mA}$, slice thickness $2.5 \mathrm{~mm}$ ) at a minimum interval of $10 \mathrm{~min}$. The study was approved by the local Ethics Committee and the informed consent was obtained from all patients.

Data obtained were analyzed independently by two radiologists to determine volumes and scores from the first and the second series, from which 12 patients present calcium. For each series, both the proposed partial voxel based method and the conventional method are used to calculate each calcium volume. Figs. 13 and 14 are two examples of the corresponding calcium 


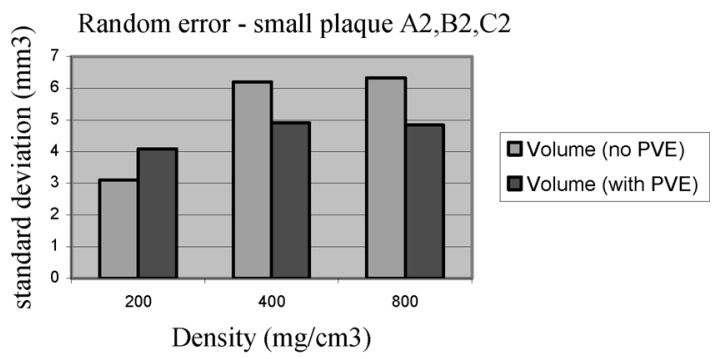

(a)

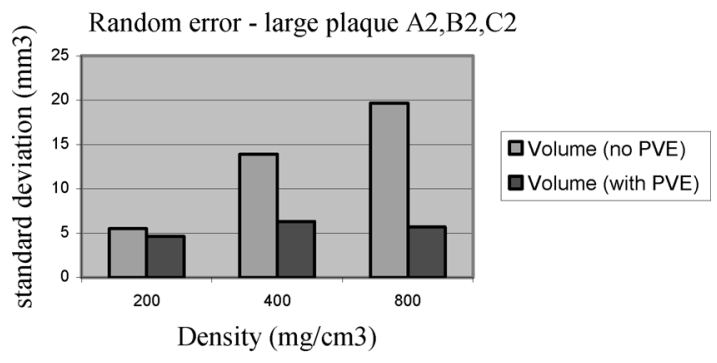

(b)

Fig. 11. Averaged reproducibility of 90 different acquisitions verses density for different plaques. (a) Small plaques. (b) Large plaques.

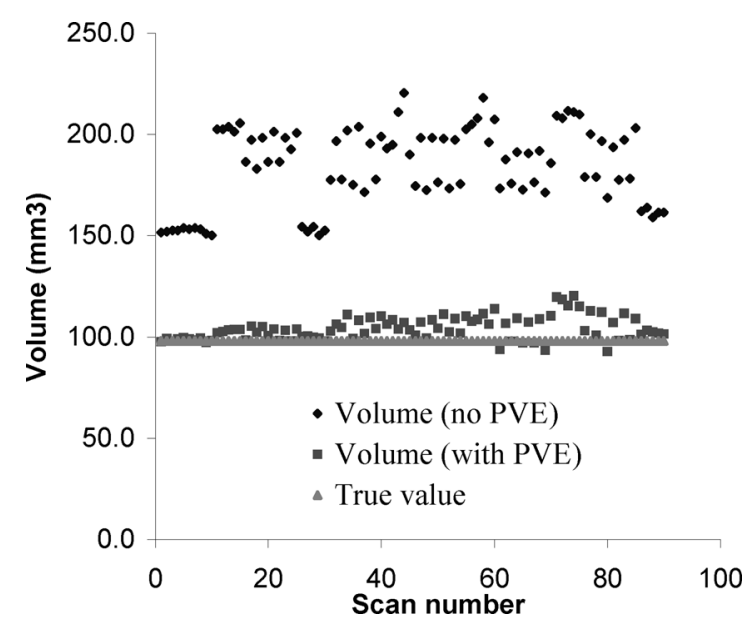

Fig. 12. Volume measurements for plaque A1 using both the proposed partial voxel based method and the conventional method.
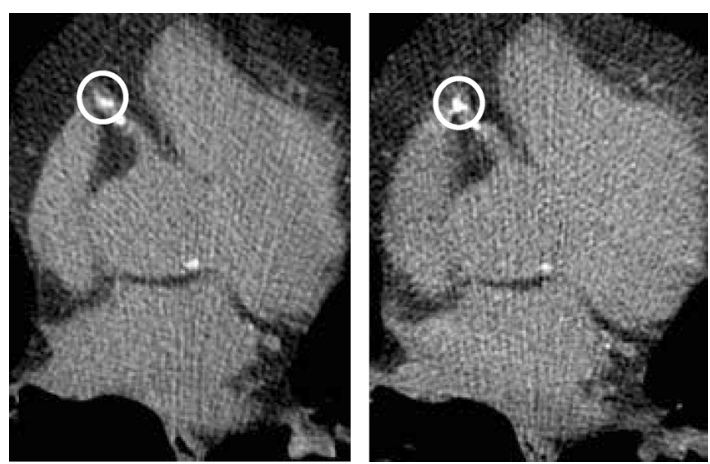

Fig. 13. Example of plaque A scanned twice using the same protocol at a minimum interval of $10 \mathrm{~min}$.

plaques in the two series. Tables III and IV show that the differences of the calculated calcium volumes between two scans are
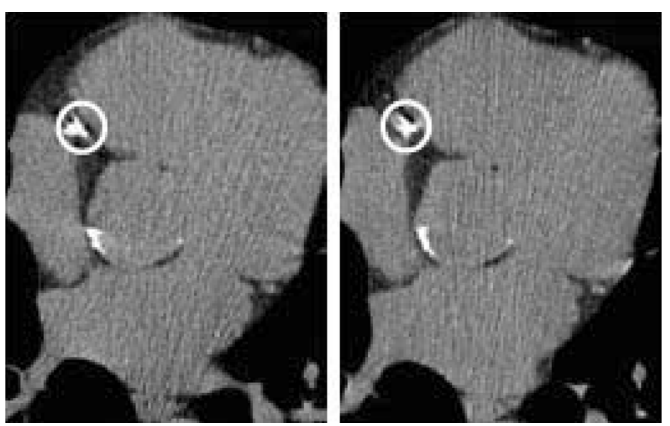

Fig. 14. Example of plaque B scanned twice using the same protocol at a minimum interval of $10 \mathrm{~min}$.

TABLE III

Volume Calculation Using the Two DifFerent Methods (Units For VOLUME IS $\mathrm{mm}^{3}$ ) FOR PLAQUe A AS Highlighted IN Fig. 13

\begin{tabular}{cccccc}
\hline \multicolumn{3}{c}{$\begin{array}{c}\text { Proposed volume } \\
\text { measurement with PVE }\end{array}$} & \multicolumn{3}{c}{$\begin{array}{c}\text { Volume measurement } \\
\text { without PVE }\end{array}$} \\
\hline \hline Vol. & Vol. & Difference & Vol. & Vol. & Difference \\
Scan 1 & Scan 2 & Difan 1 & Scan 2 & Difencec \\
\hline 66.76 & 69.9 & 3.14 & 106.69 & 126.36 & 19.67 \\
\hline
\end{tabular}

TABLE IV

Volume CALCUlations USING DifFERENT Methods (Units FOR Volume IS $\mathrm{mm}^{3}$ ) FOR PLAQUe B AS Highlighted IN FIG. 14

\begin{tabular}{cccccc}
\hline \multicolumn{2}{c}{$\begin{array}{c}\text { Proposed volume } \\
\text { measurement with PVE }\end{array}$} & \multicolumn{3}{c}{$\begin{array}{c}\text { Volume measurement } \\
\text { without PVE }\end{array}$} \\
\hline \hline Vol. & Vol. & Difference & $\begin{array}{c}\text { Vol. } \\
\text { Scan 1 }\end{array}$ & $\begin{array}{c}\text { Vol. } \\
\text { Scan 2 }\end{array}$ & Difference \\
Scan 1 & Scan 2 & & Dcan & \\
\hline 226.8 & 227.9 & 1.10 & 374.6 & 384.7 & 10.10 \\
\hline
\end{tabular}

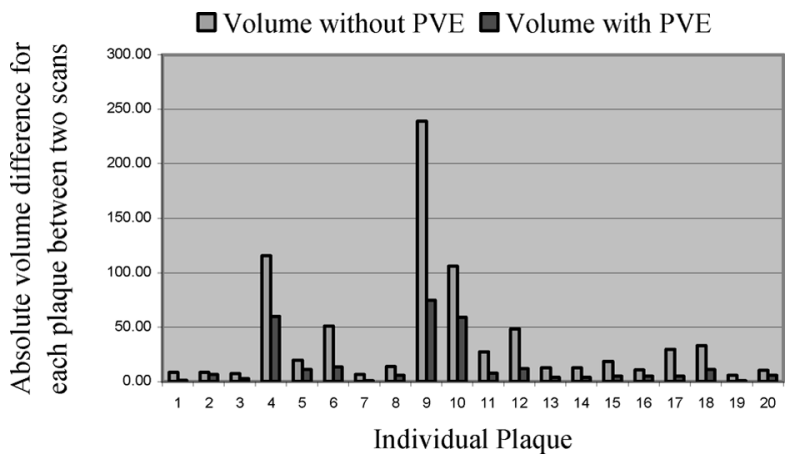

Fig. 15. Example of the absolute volume differences between two scans for 20 corresponding plaques.

$3.14 \mathrm{~mm}^{3}$ (plaque A) and $1.10 \mathrm{~mm}^{3}$ (plaque B) by using the proposed method, while using the conventional method, the differences are $19.67 \mathrm{~mm}^{3}$ (plaque A) and $10.10 \mathrm{~mm}^{3}$ (plaque B) respectively. Fig. 15 gives an example of the absolute volume difference between two scans for 20 individual plaques. It is noted that due to the cardiac motion, it is very difficult and time consuming to find one to one correspondence between the plaques in the two scans. For example, one plaque in Scan 1 might be observed to split into several pieces in Scan 2. Table V shows the total calcium volumetric measurements using the two methods. The deviation of the total calcium volume size between two series, calculated considering PVE, is about 2-3 times less than the calcium volumetric measurements without PVE. Fig. 16 
TABLE V

SUMMARY OF THE CALCIUM VOLUMETRIC MEASUREMENTS $\left(\mathrm{mm}^{3}\right.$ ) USING DIFFERENT METHODS

\begin{tabular}{cccc}
\hline Methods & $\begin{array}{c}\text { Volume of calcium } \\
\text { plaques from the first } \\
\text { scanning }\end{array}$ & $\begin{array}{c}\text { Volume of calcium } \\
\text { plaques from the } \\
\text { second scanning }\end{array}$ & Difference \\
\hline \hline $\begin{array}{c}\text { Proposed volume } \\
\text { measurements } \\
\text { with PVE }\end{array}$ & 4615.80 & 4328.18 & 287.62 \\
\hline $\begin{array}{c}\text { Volume } \\
\text { measurements } \\
\text { without PVE }\end{array}$ & 8865.00 & 8125.30 & 739.70 \\
\hline
\end{tabular}

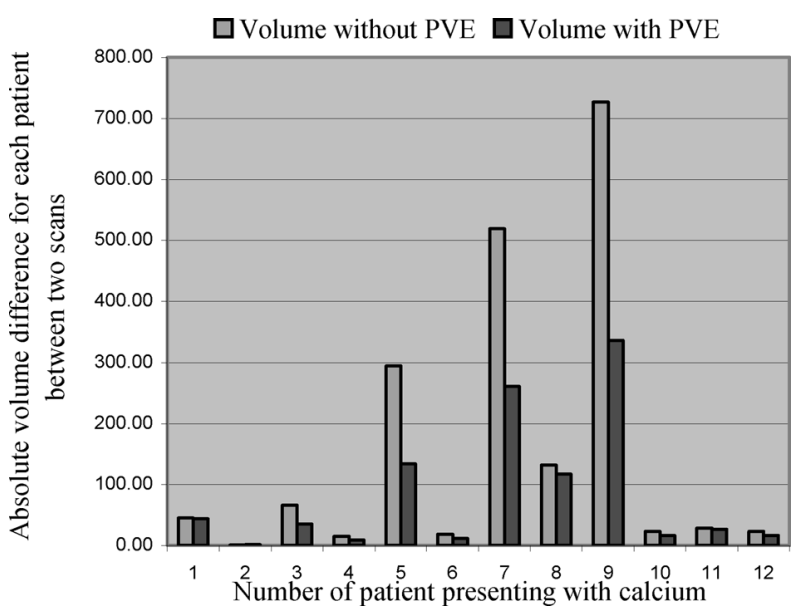

Fig. 16. Absolute volume difference between two scans for each patient having calcium.

shows the absolute volume difference between two scans for each patient It demonstrates that, with motion artifacts the proposed method gives better results in terms of reproducibility.

\section{DISCUSSION AND CONCLUSION}

Agatston et al. [12] developed a calcium scoring algorithm for EBCT that is widely used in research and clinical practice. However, reports have demonstrated that the Agatston scoring method is not suited for reliable and reproducible quantification of coronary calcified atherosclerosis plaque[13].

In this study, an attempt has been made to improve the accuracy and reproducibility of calcium volume calculation. A stochastic model based approach is presented to estimate the partial content of calcium for accurate quantification of calcium amount in the coronary arteries.

The experimental results on a QRM cardiac phantom with 90 scans using 15 different protocols and 35 patient data with repeated scans have demonstrated that the newly proposed technique gives better results compared with conventional predefined thresholding based methods in terms of accuracy and reproducibility. Significantly, the high reproducibility for the patient data demonstrates the effectiveness of the new method in the presence of motion artifacts. The deviation of the total calcium measurements is about 2-3 times less than that calculated using the traditional method. More interestingly, although the true volume of calcified plaque is obviously not available in patient data, volumes measured by the conventional method are notably up to double that of the proposed method. While, in the phantom study, the proposed method consistently calculated values which were very close to the known true values, the other method calculated values of up to double the true values. This implies an accuracy of the proposed method in the patient scans.

The proposed method has shown a statistically significant improvement over the conventional thresholding based methods when applied to the phantom and patient scans. This could result in more accurate prediction of future cardiac events and permits more accurate monitoring of the effects of risk factor modification in individual patients.

Moreover, in this study, a linear combination of tissues statistical model is used to calculate the proportion of the calcium amount. A comparison study of different PVE models and further investigation for the improvement of accuracy of calcium volume calculation are needed.

\section{REFERENCES}

[1] "From what will we die in 2020?," Lancet, vol. 349, no. 9061, 1997.

[2] T. J. Dubinsky, "Coronary artery calcification scoring," Am. J. Roentgenol., vol. 185, pp. 1540-1541, 2005.

[3] S. S. Halliburton, A. E. Stillman, and R. D. White, "Noninvasive quantification of coronary artery calcification: Methods and prognostic value," Cleve. Clin. J. Med., vol. 69, no. Suppl 3, pp. S6-11, 2002.

[4] G. Sangiorgi, J. A. Rumberger, A. Severson, W. D. Edwards, J. Gregoire, L. A. Fitzpatrick, and R. S. Schwartz, "Arterial calcification and not lumen stenosis is highly correlated with atherosclerotic plaque burden in humans: A histologic study of 723 coronary artery segments using non-decalcifying methodology," J. Am. Coll. Cardiol., vol. 31, pp. 126-133, 1998.

[5] U. J. Schoepf, C. R. Becker, B. M. Ohnesorge, and E. K. Yucel, "CT of coronary artery disease 1," Radiology, vol. 232, pp. 18-17, 2004.

[6] Y. Arad, L. A. Spadaro, K. Goodman, A. Lledo-Perez, S. Sherman, G. Lerner, and A. D. Guerci, "Predictive value of electron beam computed tomography of the coronary arteries. 19-month follow-up of 1173 asymptomatic subjects," Circulation, vol. 93, pp. 1951-1953, 1996.

[7] A. Secci, N. Wong, W. Tang, S. Wang, T. Doherty, and R. Detrano, "Electron beam computed tomographic coronary calcium as a predictor of coronary events: Comparison of two protocols," Circulation, vol. 96, pp. 1122-1129, 1997.

[8] J. A. Rumberger, D. B. Simons, and L. A. Fitzpatrick, "Coronary artery calcium area by electron-beam computed tomography and coronary atherosclerotic plaque area: A histopathologic correlative study," Circulation, vol. 92, pp. 2157-2162, 1995.

[9] S. S. Halliburton, A. E. Stillman, M. Lieber, J. M. Kasper, S. A. Kuzmiak, and R. D. White, "Potential clinical impact of variability in the measurement of coronary artery calcification with sequential MDCT," Am. J. Roentgenol., vol. 184, pp. 643-648, 2005.

[10] A. F. Kopp, B. Ohnesorge, C. Becker, S. Schröder, M. Heuschmid, A. Küttner, R. Kuzo, and C. D. Claussen, "Reproducibility and accuracy of coronary calcium measurements with multi-detector row versus electron-beam CT," Radiology, vol. 225, pp. 113-119, 2002.

[11] L. Wexler, B. Brundage, J. Crouse, R. Detrano, V. Fuster, J. Maddahi, J. Rumberger, W. Stanford, R. White, and K. Taubert, "Coronary artery calcification: Pathophysiology, epidemiology, imaging methods, and clinical implications: A statement for health professionals from the American Heart Association circulation," Circulation, vol. 94, pp. 1175-1192, 1996.

[12] A. S. Agatston and W. H. Janowitz, "Quantification of coronary artery calcium using ultrafast computed tomography," J. Am. Coll. Cardiol., vol. 15, pp. 827-832, 1990.

[13] S. Devries, C. Wolfkiel, B. Fusman, H. Bakdash, A. Ahmed, P. Levy, E. Chomka, G. Kondos, E. Zajac, and S. Rich, "Influence of age and gender on the presence of coronary calcium detected by ultrafast computed tomography," J. Amer. Coll. Cardiol., vol. 25, pp. 76-82, 1995.

[14] T. Q. Callister, B. Cooil, S. P. Raya, N. J. Lippolis, D. J. Russo, and P. Raggi, "Coronary artery disease: Improved reproducibility of calcium scoring with an electron-beam CT volumetric method," Radiology, vol 208, pp. 807-814, 1998. 
[15] H. C. Yoon, L. E. Greaser, R. Mather, S. Sinha, M. F. McNitt-Gray, and J. G. Goldin, "Coronary artery calcium: Alternate methods for accurate and reproducible quantitation," Acad. Radiol., vol. 4, pp. 666-673, 1997.

[16] C. Hong, K. T. Bae, and T. K. Pilgram, "Coronary artery calcium: Accuracy and reproducibility of measurements with multi-detector row CT: Assessment of effects of different thresholds and quantification methods," Radiology, vol. 227, pp. 795-801, 2003.

[17] M. E. Clouse, "Noninvasive screening for coronary artery disease with computed tomography is useful," Circulation, vol. 113, pp. 125-146, 2006.

[18] J. Horiguchi, H. Yamamoto, Y. Akiyama, K. Marukawa, N. Hirai, and K. Ito, "Coronary artery calcium scoring using 16-MDCT and a retrospective ECG-gating reconstruction algorithm," Am. J. Roentgenol., vol. 183, pp. 103-108, 2004.

[19] J. Dehmeshki, "An adaptive segmentation and 3-D visualisation of the lungs," Pattern Recognit. Lett., vol. 20, pp. 919-926, 1999.
[20] D. H. Laidlaw, K. W. Fleischer, and A. H. Barr, "Partial-volume Bayesian classification of material mixtures in MR volume data using voxel histograms," IEEE Trans. Med. Imag., vol. 17, no. 1, pp. 74-86, Feb. 1998.

[21] K. V. Leemput, F. Maes, D. Vandermeulen, and P. Suetens, "A unifying framework for partial volume segmentation of brain MR images," IEEE Trans. Med. Imag., vol. 22, no. 1, pp. 105-117, Jan. 2003.

[22] Y. Du, B. M. W. Tsui, and E. C. Frey, "Partial volume effect compensation for quantitative brain SPECT imaging," IEEE Trans. Med. Imag., vol. 24, no. 8, pp. 969-976, Aug. 2005.

[23] P. Santago and H. D. Gage, "Statistical models of partial volume effect," IEEE Trans. Med. Imag., vol. 4, no. 11, pp. 1531-1539, Nov. 1995.

[24] R. C. Gonzalez and R. E. Woods, Digital Image Processing, 2nd ed. New York: Addison Wesley, 2002.

[25] S. Z. Li, Markov Random Field Modeling in Computer Vision. New York: Springer-Verlag, 1995. 